\title{
Toward the detection of intraplaque hemorrhage in carotid artery lesions using photoacoustic imaging
}

\author{
Mustafa Umit Arabul \\ Maarten Heres \\ Marcel C. M. Rutten \\ Marc R. van Sambeek \\ Frans N. van de Vosse \\ Richard G. P. Lopata
}




\title{
Toward the detection of intraplaque hemorrhage in carotid artery lesions using photoacoustic imaging
}

\author{
Mustafa Umit Arabul, ${ }^{\mathrm{a}, *}$ Maarten Heres, ${ }^{\mathrm{a}}$ Marcel C. M. Rutten, ${ }^{\mathrm{a}}$ Marc R. van Sambeek, \\ Frans N. van de Vosse, ${ }^{a}$ and Richard G. P. Lopata ${ }^{a}$ \\ ${ }^{a}$ Eindhoven University of Technology, Department of Biomedical Engineering, Cardiovascular Biomechanics Group, De Zaale, \\ Eindhoven 5612 AJ, The Netherlands \\ ${ }^{\mathrm{b} C}$ Catharina Hospital Eindhoven, Department of Vascular Surgery, Michelangelolaan 2, Eindhoven 5623 EJ, The Netherlands
}

\begin{abstract}
Photoacoustic imaging (PAI) may have the ability to reveal the composition and the anatomical structure of carotid plaques, which determines its mechanical properties and vulnerability. We used PAI and plane wave ultrasound (PUS) imaging to obtain three-dimensional (3-D) images of endarterectomy samples ex vivo and compared the results with histology to investigate the potential of PAl-based identification of intraplaque hemorrhage. Seven carotid plaque samples were obtained from patients undergoing carotid endarterectomy and imaged with a fully integrated hand-held photoacoustic (PA) probe, consisting of a pulsed diode laser $\left(t_{\text {pulse }}=130 \mathrm{~ns}, E_{\text {pulse }}=1 \mathrm{~mJ}, \lambda=808 \mathrm{~nm}\right)$ and a linear array transducer $\left(f_{c}=7.5 \mathrm{MHz}\right)$. The samples were rotated 360 deg with 10 deg steps, and data were spatially compounded to obtain complete 3-D images of the plaques. Areas of high absorption in the 3-D datasets were identified and compared to histological data of the plaques. Data in six out of seven endarterectomy samples revealed the presence of intraplaque hemorrhages that were not visible in the PUS images. Due to the noninvasive nature of PAI, this ex vivo study may elucidate preclinical studies toward the in vivo, noninvasive, vulnerability assessment of the atherosclerotic carotid plaque. @ 2016 Society of Photo-Optical Instrumentation Engineers (SPIE) [DOI: 10.1117/1.JBO.22.4.041010]
\end{abstract}

Keywords: photoacoustics; plane wave ultrasound; spatial compounding; carotid plaques; intraplaque hemorrhage.

Paper 160568SSRR received Aug. 24, 2016; accepted for publication Dec. 1, 2016; published online Dec. $23,2016$.

\section{Introduction}

The abrupt rupture of an atherosclerotic carotid plaque is one of the main causes of stroke and stroke-induced death. ${ }^{1,2}$ Current practice to prevent stroke in patients with a significant carotid stenosis is the removal of the plaque during an endarterectomy procedure. The current decision-making procedure for patients with atherosclerotic plaques to undergo an endarterectomy is based on the severity of stenosis. However, a study by Rothwell and Warlow ${ }^{3}$ has shown that only $16 \%$ of the patients that underwent endarterectomy, based on the degree of the stenosis of their carotid plaque (70\% to $99 \%)$, were really at risk to suffer from stroke in a 5-year period. Although the complete rupture mechanism is not resolved yet, there is strong evidence supporting the hypothesis of plaque composition playing a key role in plaque rupture rather than the degree of stenosis. ${ }^{4,5}$

Retrospective studies have demonstrated that the common features of the risk of plaque rupture are the existence of a large lipid necrotic core, a thin fibrous cap over the core, and intraplaque hemorrhages ${ }^{6-8}$ However, common clinical practice is still based on the estimation of the degree of stenosis based on duplex ultrasound. In case ultrasonography was insufficient due to excessive calcifications in the plaque, additional magnetic resonance imaging (MRI) or computed tomography angiography (CTA) scans were often required. Nevertheless, the studies performed with $\mathrm{MRI}^{9-11}$ or CTA ${ }^{12,13}$ showed moderate sensitivity $(60 \%$ to $92 \%)$ and specificity $(74 \%)$ in detecting the plaque morphology.
Despite all the advantages of the current imaging modalities, the unmet need is a noninvasive approach suitable for patient follow-up that can distinguish between different plaque components. ${ }^{4,14}$ The wavelength-specific optical absorption differences of distinct constituents of plaque might be advantageous for creating contrast for imaging. ${ }^{15,16}$ In photoacoustics (PAs), the tissue is illuminated with short-pulsed laser light, and ultrasound is generated locally inside the tissue wherever the optical energy is absorbed. Since the scattering of acoustic waves is negligible for the soft tissue, imaging depth can reach up to $3 \mathrm{~cm}$ with large area illumination. ${ }^{17}$ Combining the optical contrast with ultrasonic resolution and imaging depth, photoacoustic imaging (PAI) may outperform MRI and CTA for plaque assessment, with a comparably low cost and practical applicability similar to ultrasound.

In early studies, the applicability of intravascular photoacoustic (IVPA) imaging was investigated on phantoms and also on ex vivo rabbit arteries. ${ }^{18}$ Results showed that the use of IVPA accompanied by intravascular ultrasound may provide morphological information in the assessment of atherosclerotic plaques. Moreover, spectroscopic IVPA provides an additional dimension of assessment depending on different constituents. ${ }^{19}$ The capability of IVPA on detecting labeled macrophages as well as lipids embedded in polyvinyl alcohol (PVA) vessel phantoms was shown. ${ }^{20-22}$ Successful differentiation of distinct lipid types have been reported on a PVA phantom ${ }^{23}$ and on human coronary samples ${ }^{24}$ using multispectral IVPA. The feasibility of intravascular photoacoustic tomography (IVPAT) for imaging 
lipid accumulation over time (25 weeks follow-up study) was shown in the aortas of rabbits that were on a high cholesterol diet. ${ }^{25}$ Recently, an ex vivo study was reported using IVPA for high speed imaging of lipid pools in carotid plaques. ${ }^{26}$

IVPA carries huge potential, especially for coronary plaques where catheterization is inevitable. However, a catheter-based solution is less suitable for carotid plaque assessment before deciding on treatment. Furthermore, the superficial nature of the carotid artery ( 1 to $3 \mathrm{~cm}$ ) makes it an attractive application for noninvasive PA studies. Therefore, an in vivo approach of noninvasive PAs combined with ultrasound is regarded as a more accessible method. There are in vivo studies using a multispectral tomographic approach using a concave curved array around the neck, ${ }^{27}$ internal illumination via the pharynx, ${ }^{28}$ and external acquisition from the surface of the skin. Both studies presented promising phantom experiments; however, the specificity of the in vivo measurements was insufficient for drawing any conclusions.

Aforementioned PAI studies mostly focused on lipid detection and yet, the in vivo results are still sparse and often lack proper validation. In addition to the lipid in the plaque, intraplaque hemorrhages are fundamental indicators of plaque rupture. ${ }^{29}$ Findings of medical studies in the last decade led the interest toward intraplaque hemorrhage and vasa vasorum density as better risk predictors of plaque vulnerability. ${ }^{30}$ Although there are a few studies that aim to detect intraplaque hemorrhages using MRI ${ }^{10,31}$ or CTA, ${ }^{32}$ it was disregarded by the previous PA studies.

The aim of this study is to demonstrate and validate the ability of PAI of carotid plaques, specifically targeting intraplaque hemorrhage and neo-vascularization. To our knowledge, intraplaque hemorrhage was imaged for the first time in human tissue samples using PAI. The uncertainty of the optical energy reaching the plaque and the morphological complexity of the plaque were the two major challenges of the in vivo experiments. To identify the challenges of in vivo PAI of the carotid artery, the PA response of the human carotid plaque should be understood thoroughly. Therefore, an experimental setup, which allows complete rotation of the sample and creates a better quality three-dimensional (3-D) imaging using multiangle spatial compounding, was designed to investigate the PA signals of the human carotid samples ex vivo. In this study, we used an integrated PA probe ${ }^{33}$ and combined PA and plane wave ultrasound (PUS) images to obtain 3-D morphology of the samples. The wavelength of the probe was chosen to be $808 \mathrm{~nm}$ to obtain optimal absorption by the blood at the focal depth $(\sim 20 \mathrm{~mm})$ of the ultrasound transducer. For validation, we compared the overlaid 3-D PA and PUS images to the ground truth, i.e., the histology.

\section{Methods}

\subsection{Sample Preparation and Experimental Setup}

The endarterectomy samples were obtained from the Department of Vascular Surgery of the Catharina Hospital Eindhoven. All patients $(n=7)$ had ipsilateral neurological symptoms within the two weeks prior to the surgery; therefore, the samples are assumed to be vulnerable/ruptured plaques. The study was approved by the local ethics committee and all patients gave their informed consent. The carotid endarterectomy was performed using a method similar to the one described by Wijeyaratne et al. ${ }^{34}$ to extract the plaques as intact as possible. If the plaque was fully intact, it was transferred to the laboratory in a phosphate-buffered saline (PBS) solution. The plaque samples were flushed with PBS to remove blood clots from the outer surface of the media layer and inner surface of the intima. Washing out excess blood reduced possible clutter artifacts during PAI.

After cleaning the plaque sample, plastic cannulas were attached to both ends of the carotid artery to enable rigid mounting of the sample in the PA/PUS imaging setup (Fig. 1). The setup was designed to perform imaging during rigid rotation of vessel samples with $10 \mathrm{deg}$ steps. ${ }^{35}$ A motorized 3-D stage (M-403.2DG, Physik Instrumente, Germany) was mounted to the setup to precisely adjust the position of the PAI probe.

\subsection{Hand-Held Photoacoustic Probe}

For both PA and PUS imaging, a hand-held PA probe was used. This probe has an integrated diode laser system $\left(t_{\text {pulse }}=130 \mathrm{~ns}\right.$, $E_{\text {pulse }}=1 \mathrm{~mJ}, \lambda=808 \mathrm{~nm}$, QUANTEL, France, OSRAM, Germany, SILIOS, France) and a linear array ultrasound transducer (SL3323, $f_{c}=7.5 \mathrm{MHz}$, ESAOTE Europe, Maastricht, The Netherlands) for the acquisition. The pulse repetition rate (PRF) of the laser and the acquisition system is software limited to $2 \mathrm{kHz}$ due to the laser safety regulations. The point-spread function measurements of the system revealed that the system has $0.5-\mathrm{mm}$ mean resolution in the lateral direction and $0.28 \mathrm{~mm}$ in the axial direction. ${ }^{33}$ The detailed operation and specifications of the system and the hand-held probe (developed by the FULLPHASE Consortium) were described by Daoudi et al. ${ }^{33}$

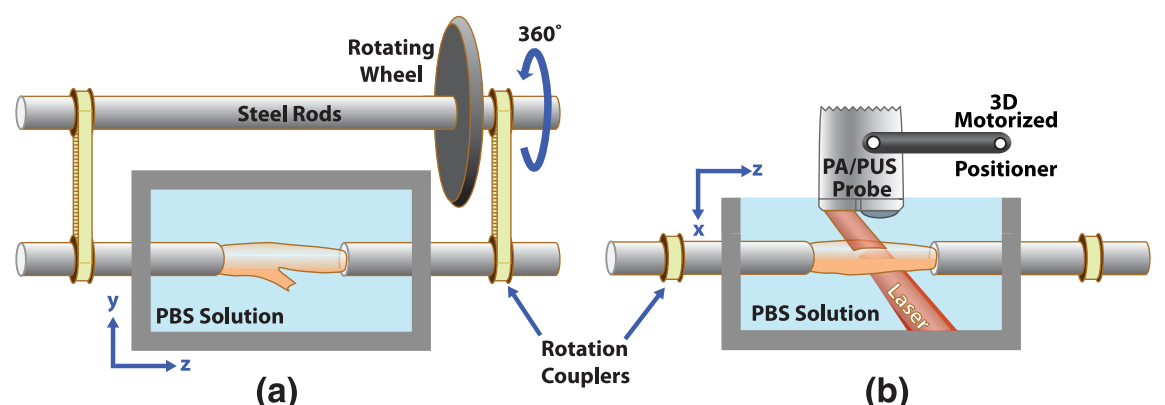

Fig. 1 Illustration of the experimental setup (a) from the top view and (b) from the side view. The sample was immersed into phosphate-buffered-saline (PBS) solution. The imaging probe was positioned in the cross-section of the sample for PA imaging. 


\subsection{Scanning Protocol}

The probe was positioned in the transverse plane of the sample, and the plaque was mechanically scanned along its longitudinal axis. PUS data were recorded at $10-\mathrm{Hz}$ PRF during forward scanning and PA data were recorded at $100-\mathrm{Hz}$ PRF during backward scanning. The mechanical scanning speed of the 3-D stage was $1.0 \mathrm{~mm} / \mathrm{s}$; hence, the distances between two consecutive frames were $100 \mu \mathrm{m}$ for PUS and $10 \mu \mathrm{m}$ for PA acquisitions. A higher PRF was preferred in PA acquisition to allow more averaging in postprocessing to increase the signal-tonoise ratio (SNR). After obtaining the data for both modalities, the sample was rotated by $10 \mathrm{deg}$ and the measurements were repeated for all 36 angles.

\subsection{Postprocessing}

During acquisition, the raw radio frequency (RF) data were acquired with a sampling rate of $50 \mathrm{MHz}$ and stored. Data were processed offline using MATLAB 2014b (The MathWorks, Natick, Massachusetts). The data were denoised with a series of digital filters, a DC blocking, a moving average $(N=20)$ for reducing the random electrical noise, and a bandpass filter $\left(f_{\text {low }}=0.8 \mathrm{MHz} f_{\text {high-PA }}=5.5 \mathrm{MHz}, f_{\text {high-PUS }}=15 \mathrm{MHz}\right)$ for suppressing the frequency content out of the acoustic bandwidth of the probe.

Beamformed RF data were reconstructed using the conventional delay-and-sum reconstruction method. After obtaining reconstructed ultrasound and PA acquisitions, data were rotated in reference to the position of the sample obtained at 0 deg. Next, data from all angles were spatially compounded into one 3-D volume image to obtain a higher SNR and contrast-to-noise ratio. Spatial compounding provided geometry and morphology (mostly calcification) independent imaging performance and lead a high resolution and contrast for the complete plaque. Finally, PA and PUS images were overlaid by assigning a transparency to PA image depending on its intensity value.

\subsection{Histology Comparison}

After PA/PUS imaging, five of seven samples (one sample was fractured during the snap-freeze process and one sample was used for other purposes) were fixated in glycol (Tissue-Tek O.C.T., Sakura Finetek Europe, The Netherlands) solution and snap-frozen in liquid nitrogen. Each frozen sample was mounted to a cryotome (HM550, Thermo Scientific) to obtain histology cross-sections. The samples were sliced with $10-\mu \mathrm{m}$ thickness. Since intraplaque hemorrhages were clearly identifiable in the white background of the frozen glycol solution, no additional staining needed to be performed. Instead, for each cross-sectional slice, an image was recorded by a digital camera. A total of 2000 images were obtained from each plaque sample. This enabled a complete geometrical inspection, by comparing the slices to the PUS data.

Next, four samples that had a positive indication of hemorrhage in the PA images were selected for comparison with histology. The intraplaque hemorrhages were identified in the histology pictures, and five distinct regions in each plaque were selected for comparison. The corresponding crosssectional PUS data were identified, based on the similarity in geometry. Next, the same regions with hemorrhage in histology were selected in the PA/PUS images, and the corresponding PA intensities were compared to the PA data of the remaining vessel area.

\section{Results}

The 3-D rendered volume images of PUS reveal the anatomy and shape of the plaque, clearly resembling the shape in the pictures of the plaques (Fig. 2), which were available for five cases. The pictures of the two other samples were absent due to the fact that the intact geometry comparison was initially unintended and pictures were not taken. As expected, there is no contrast between the soft plaque content and the vessel wall. Conversely, the PA images solely provide the absorbing regions inside the plaque, i.e., the regions filled with blood (Fig. 2). The overlaid 3-D volumes provide an overview of the samples;
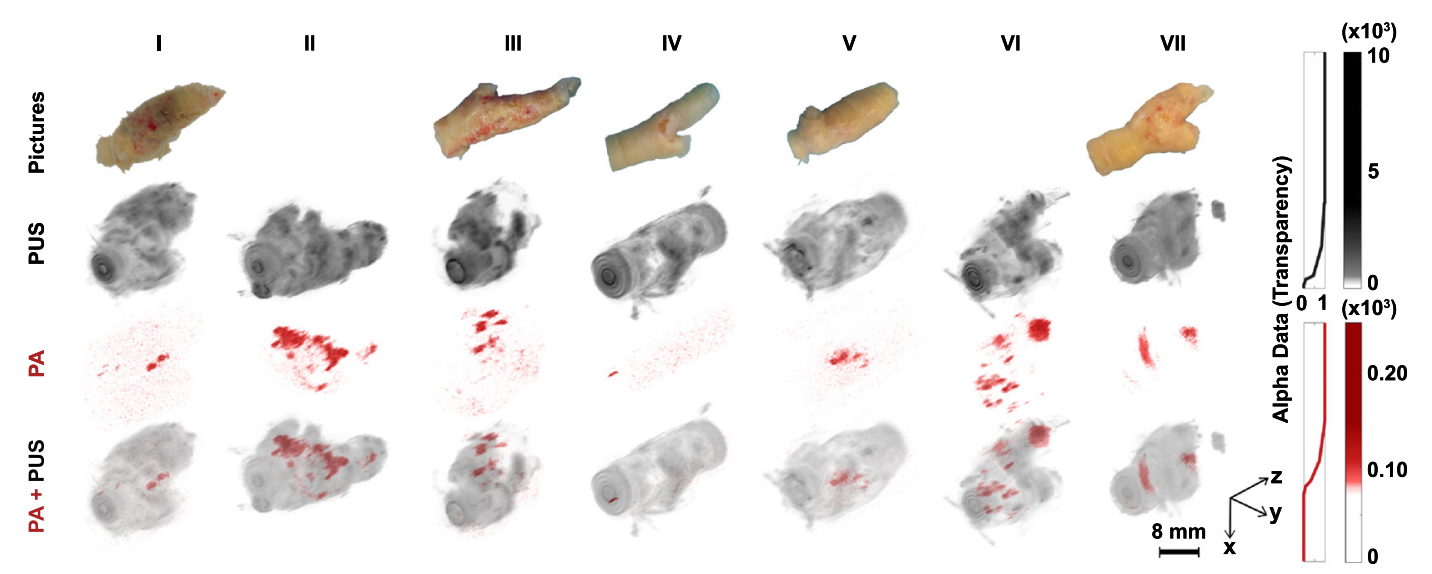

Fig. 2 Imaging results for all plaque samples (I to VII). Pictures of the plaques are shown in the first row when available. The 3-D plane wave ultrasound (PUS, second row), photoacoustic (PA, third row), and the overlaid PA/PUS images (bottom row) of the human carotid plaques are shown. Darker regions in the grayscale represent the higher echogenicity in pulse-echo ultrasound. The red regions show the PA signals, which correspond to a higher absorption of optical energy, i.e., higher concentration of blood content. Overlaid PA/PUS images with transparency indicate the possible intraplaque hemorrhage location in these atherosclerotic plaque samples. 


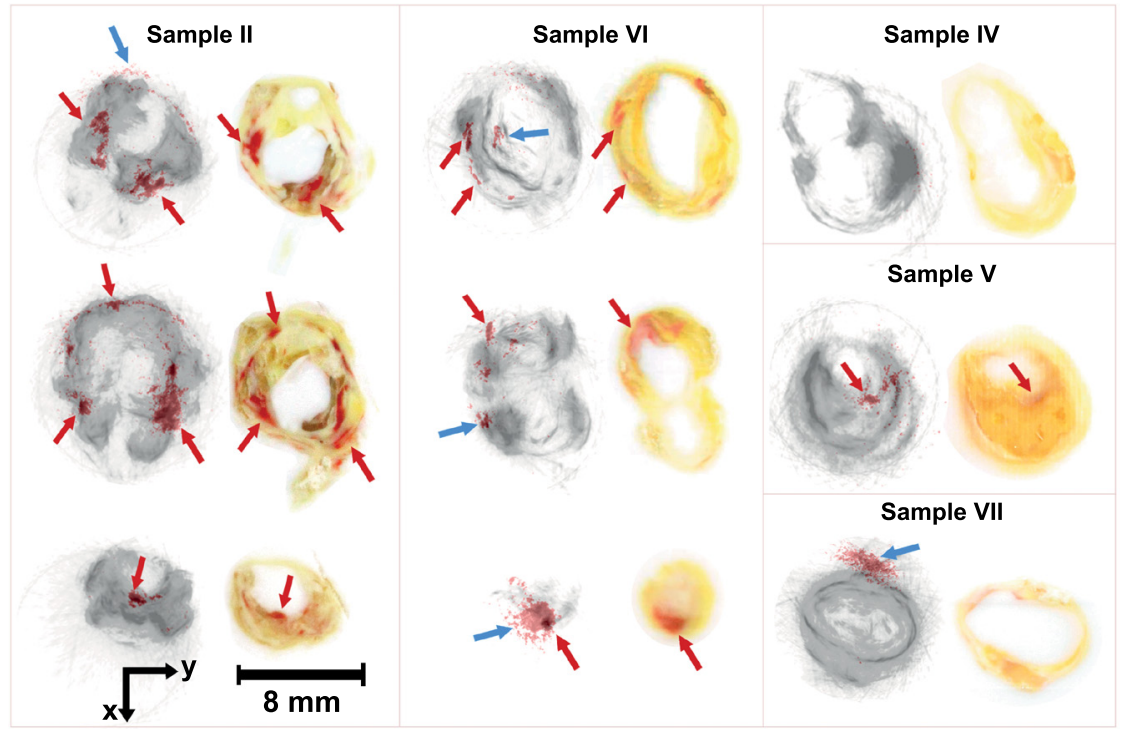

Fig. 3 Pictures of histology sections and the corresponding cross-sections in overlaid PA-PUS images of five plaques. Gray color represents the ultrasound signals and overlaid red colors represent the PA signals. The red arrows indicate the blood content inside the plaques in the histology pictures and the corresponding PA signals in overlaid PA/PUS images. Blue arrows indicate the clutter artifacts and regions with overestimation of the PA signals.

however, for a more elaborate comparison, the transverse views need to be compared to the histology (see Fig. 3).

In the histologic cross-sections, the yellow regions are the vessel wall, whereas the dark red areas are hemorrhages. The darker (brownish-yellow) color represents the calcified area in the plaque, and the light yellow area is in this case the lipid pool, which is not really distinguishable from the wall in these images (see Fig. 3). However, since targeting lipid was not an aim of this study, no additional histological analysis on lipid content was performed.

Qualitative comparison of overlaid PA/PUS images to histology reveals one to one correspondence between the two (see the red arrows in Fig. 3). These images confirm that the high signal intensity in PA images indicates regions with high blood content, most likely intraplaque hemorrhage. There are additional regions located outside of the vessel with high intensity signals (see the blue arrows in Fig. 3) for some of the cross-sections. Those signals were identified as clutter artifacts and were recognized based on the geometry provided by PUS images. The quantification of the severity of the clutter was not performed, and in the scope of this study, the clutter artifacts were assumed not to deteriorate the actual PA signals significantly.

The quantitative comparison of the segmented regions with hemorrhage versus the remainder of the vessel area supports the objective of this study. The PA signal intensities in the segmented area were significantly higher (one way ANOVA, $p<0.001$ ), whereas the PUS gray values were not (Fig. 4). An additional correlation analysis between US and PA images was performed to show the independence of the two modalities. There was no correlation in the hemorrhage segments
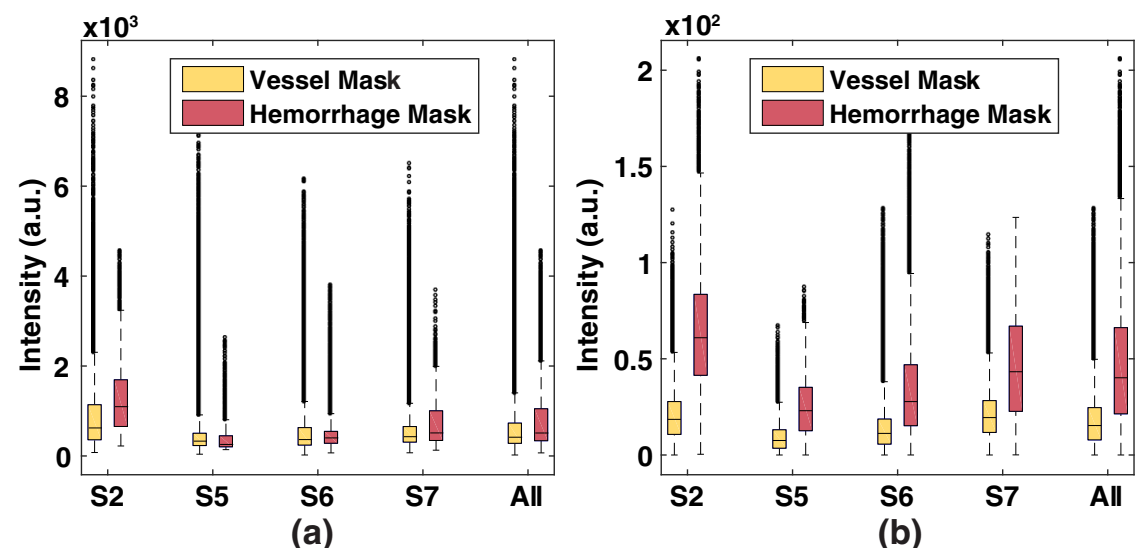

Fig. 4 Statistical comparison of the intensities in the segmented regions of hemorrhages (a) boxplots of the gray values of ultrasound images in regions with hemorrhage (red) and those found in the surrounding tissue region (yellow), for samples II, V, VI and VII; (b) boxplots of the photoacoustic signal measured in hemorrhage segments (red) versus those detected in the surrounding tissue (yellow). The black circles are data points that are considered outliers of the statistical distribution. 
$\left(R^{2}=7.01 \pm 8.05 \%\right)$ and the rest of the vessel segments $\left(R^{2}=2.10 \pm 4.22 \%\right)$ for all the selected frames in the histology comparison.

\section{Discussion}

In this study, we have demonstrated the feasibility of PAI to locate intraplaque hemorrhages (any intraplaque blood pools) in atherosclerotic carotid plaques as a first step toward noninvasive plaque characterization. We used a hand-held PA probe with integrated diode laser and a portable medical ultrasound system. Signals corresponding to blood-filled regions, which can be extensions of the vasculature in the plaque, intraplaque hemorrhage, or the result of trauma inflicted on the medial vascularization, were found in the PA images with high contrast with respect to the wall, whereas these regions were not distinguishable in the PUS images. The detection of intraplaque hemorrhage in carotid plaques is the novelty of this study and promising toward in vivo preclinical studies regarding carotid plaque vulnerability assessment.

The histologic cross-sections show one to one correspondence with the overlaid PA and ultrasound images. While ultrasound images reveal the shape of the vessel, PA images show where the intraplaque hemorrhage is located (Fig. 2). The lack of PA signals in the rest of the plaque components, such as lipid, calcification, and vessel wall, proves that the optical absorption of those constituents at $808 \mathrm{~nm}$ illumination is not sufficient to create detectable PA signals. Moreover, the histology-based manual segmentation validated that the PA intensities were significantly higher in the segmented regions.

The overlaid PA images contain some clutter artifacts (Fig. 2) due to optical energy deposition outside the imaging plane or multiple reflections from calcifications (highly echogenic) in the vicinity of the blood content. By adopting recently developed clutter reduction methods, we may benefit from the pulsation of the carotid artery in vivo to reduce the clutter. ${ }^{36}$ Furthermore, a priori ultrasound data can be used to reduce the reflection clutter. ${ }^{37}$

The PA/PUS images were obtained in a controlled setting using a tomographic approach by rotating the samples, which provides a complete cross-sectional view. This approach also overcomes the limited field-of-view in the presence of excessive calcifications. Obviously, this is not a method designed for, or feasible in, in vivo applications, but purely for preclinical validation of the PA/PUS techniques. The tomographic approach in this study could be partially translated to in vivo scanning via the development of novel, optimal multiangle, in vivo imaging methods.

The use of integrated diode laser lowers the available energy of the imaging system; however, the limiting factor for the maximum permeable energy (MPE) of the laser is the medical laser device standards (IEC/EN 60601-2-22 and IEC 608251) considering its future clinical use. The illumination scheme was optimized for highest optical flux at the focal depth of the ultrasound transducer $(\sim 20 \mathrm{~mm})$. In the next prototypes, energy could be increased within the clinical safety limits. Nevertheless, in the scope of this study, the 1-mJ pulse energy was sufficient to provide the images of plaque samples in the $e x$ vivo conditions.

The ex vivo imaging protocol developed in this study will contribute much needed, ground truth knowledge to overcome in vivo problems in PA imaging of the carotid plaques. The imaging depth of the PA system used is in the range of the anatomical depth of carotid arteries. However, the imaging system is not likely to achieve similar performances for patients with a high body-mass index. For instance, the peripheral vessels that are located between the skin and carotid artery and the presence of luminal blood will reduce the optical power that reaches the plaque. However, the multiangle spatial compounding compensates the signal loss due to luminal blood absorption. Despite the in vivo challenges, the experimental method used in this study can provide complementary information to compare the preoperative in vivo images to the postoperative, ex vivo images of both PA and PUS. Handling those challenges within laser safety limits will require the development of novel techniques based on the ground truth knowledge. Therefore, the ability to detect intraplaque hemorrhages while keeping the plaque intact will enable further research on increasing the SNR of the system in the presence of surrounding tissue and aforementioned in vivo conditions. Similarly, the presence of luminal blood besides the intraplaque blood will require a technique to distinguish between the two. Using the high temporal resolution of PA/PUS, and the fact that luminal blood will move with a high speed whereas thrombus will not, may provide a solution to this problem. Moreover, the absorption spectrum of thrombus slightly deviates from hemoglobin, which may be beneficial for PA spectroscopic techniques using multiple wavelengths to distinguish between fresh blood and thrombus. Such a multispectral approach can also be used to reveal additional morphological features of the plaques.

\section{Conclusion}

In summary, PA and PUS imaging of intraplaque hemorrhages in human carotid plaques were performed as a first step toward in vivo imaging. A fully integrated PA probe was used externally, and spatial compounding of acquisitions from different angular positions was performed to obtain full 3-D images of the samples with better image quality. Since intraplaque hemorrhage is considered to be one of the indicators of plaque vulnerability, this study showed that PAI can be used in vulnerability assessment of atherosclerotic carotid plaques. Future work, at higher penetration depths, will demonstrate its clinical merit.

To obtain the complete morphology (lipid pool, fibrous cap, and intraplaque hemorrhage) of a plaque, multispectral PA is necessary. We performed the measurements with single wavelength illumination and in isolated conditions to investigate the possibility of PAI for intraplaque hemorrhage detection. In future studies, the PA imaging performance will be further investigated in the presence of luminal blood, the pulsation of the vessel, ${ }^{38}$ and surrounding tissue. Incrementally approaching to more realistic conditions will ease the development of novel solutions to the challenges in PA imaging in vivo. Eventually, the ability to differentiate between rupture-prone and stable plaques will contribute to clinical decision making in vascular surgery. A reduction in overtreatment of the stable plaques will lower the burden on the patient and will reduce the risks and costs involved. Furthermore, prevention of stroke or other complications, by early detection of unstable plaques, will reduce the mortality and morbidity rates and, most importantly, increase the quality of life in aging societies.

\section{Disclosures}

Hereby, we certify that there is no financial interest in this study to disclose. 


\section{Acknowledgments}

This study is funded by the European Community's Seventh Framework Programme (FP7/2007-2013) under grant agreement no. 318067.

\section{References}

1. S. Chaturvedi et al., "Carotid endarterectomy—an evidence-based review: report of the therapeutics and technology assessment subcommittee of the american academy of neurology," Neurology 65, 794-801 (2005).

2. D. Mozaffarian et al., "Heart disease and stroke statistics—2015 update: a report from the american heart association," Circulation 131(4), e29-e322 (2015).

3. P. M. Rothwell and C. P. Warlow, "Prediction of benefit from carotid endarterectomy in individual patients: a risk-modelling study," Lancet 353(9170), 2105-2110 (1999).

4. W. Casscells, M. Naghavi, and J. T. Willerson, "Vulnerable atherosclerotic plaque: a multifocal disease," Circulation 107(16), 2072-2075 (2003).

5. R. A. Knox, P. J. Breslau, and D. E. Strandness, "A simple parameter for accurate detection of severe carotid disease," Br. J. Surg. 69(4), 230-233 (1982).

6. M. J. Davies et al., "Risk of thrombosis in human atherosclerotic plaques: role of extracellular lipid, macrophage, and smooth muscle cell content," Heart 69(5), 377-381 (1993).

7. R. Virmani et al., "Pathology of the vulnerable plaque," J. Am. Coll. Cardiol. 47(8 Suppl), C13-C18 (2006).

8. A. V. Finn et al., "Concept of vulnerable/unstable plaque," Arterioscler. Thromb. Vasc. Biol. 30, 1282-1292 (2010).

9. S. E. Clarke et al., "Quantitative assessment of carotid plaque composition using multicontrast MRI and registered histology," Magn. Reson. Med. 50(6), 1199-1208 (2003).

10. B. Chu et al., "Hemorrhage in the atherosclerotic carotid plaque: a highresolution MRI study," Stroke 35(5), 1079-1084 (2004).

11. S. Fabiano et al., "High-resolution multicontrast-weighted MR imaging from human carotid endarterectomy specimens to assess carotid plaque components," Eur. Radiol. 18(12), 2912-2921 (2008).

12. L. J. Walker et al., "Computed tomography angiography for the evaluation of carotid atherosclerotic plaque: correlation with histopathology of endarterectomy specimens," Stroke 33(4), 977-981 (2002).

13. T. T. De Weert et al., "In vivo characterization and quantification of atherosclerotic carotid plaque components with multidetector computed tomography and histopathological correlation," Arterioscler. Thromb. Vasc. Biol. 26(10), 2366-2372 (2006).

14. M. Naghavi et al., "From vulnerable plaque to vulnerable patient: a call for new definitions and risk assessment strategies: Part I," Circulation 108, 1664-1672 (2003).

15. M. J. C. Van Gemert et al., "Optical properties of human blood vessel wall and plaque," Lasers Surg. Med. 5, 235-237 (1985).

16. S. A. Prahl, M. J. C. V. Gemert, and A. J. Welch, "Determining the optical properties of turbid media," Opt. Soc. Am. 32(4), 559-568 (1993).

17. L. V. Wang and S. Hu, "Photoacoustic tomography: in vivo imaging from organelles to organs," Science 335, 1458-1462 (2012).

18. S. Sethuraman et al., "Intravascular photoacoustic imaging using an IVUS imaging catheter," IEEE Trans. Ultrasonics Ferroelect. Freq. Contr. 54, 978-986 (2007).

19. S. Sethuraman et al., "Spectroscopic intravascular photoacoustic imaging to differentiate atherosclerotic plaques," Opt. Express 16, 33623367 (2008).

20. B. Wang et al., "Plasmonic intravascular photoacoustic imaging for detection of macrophages in atherosclerotic plaques," Nano Lett. 9, 2212-2217 (2009).

21. B. Wang et al., "Intravascular photoacoustic imaging," IEEE J. Select. Top Quantum Electron. 16, 588-599 (2010).

22. B. Wang et al., "Intravascular photoacoustic imaging of lipid in atherosclerotic plaques in the presence of luminal blood," Opt. Lett. 37, 12441246 (2012).

23. M. Wu et al., "Specific imaging of atherosclerotic plaque lipids with two-wavelength intravascular photoacoustics," Biomed. Opt. Express 6(9), 3276-3286 (2015).
24. K. Jansen et al., "Photoacoustic imaging of human coronary atherosclerosis in two spectral bands," Photoacoustics 2, 12-20 (2014).

25. J. Zhang et al., "Characterization of lipid-rich aortic plaques by intravascular photoacoustic tomography," J. Am. Coll. Cardiol. 64(4), 385-390 (2014).

26. Y. Cao et al., "High-sensitivity intravascular photoacoustic imaging of lipid-laden plaque with a collinear catheter design," Sci. Rep. 6, 25236 (2016).

27. A. Dima and V. Ntziachristos, "Non-invasive carotid imaging using optoacoustic tomography," Opt. Express 20, 25044-25057 (2012).

28. P. Kruizinga et al., "Photoacoustic imaging of carotid artery atherosclerosis," J. Biomed. Opt. 19, 110504 (2014).

29. H. Sillesen and T. Nielsen, "Clinical significance of intraplaque hemorrhage in carotid artery disease," J. Neuroimaging 8(1), 15-19 (1998).

30. P. R. Moreno et al., "Plaque neovascularization is increased in ruptured atherosclerotic lesions of human aorta: implications for plaque vulnerability," Circulation 110(14), 2032-2038 (2004).

31. J. Scott McNally et al., "Carotid MRI detection of intraplaque hemorrhage at 3T and 1.5T," J. Neuroimaging 25(3), 390-396 (2015).

32. J. M. U-King-Im et al., "Characterization of carotid plaque hemorrhage: a CT angiography and MR intraplaque hemorrhage study," Stroke 41(8), 1623-1629 (2010).

33. K. Daoudi et al., "Handheld probe integrating laser diode and ultrasound transducer array for ultrasound/photoacoustic dual modality imaging," Opt. Express 22, 26365-26374 (2014).

34. S. Wijeyaratne, C. Abbott, and M. Gough, "A modification to the standard technique for carotid endarterectomy allowing removal of intact endarterectomy specimens: implications for research and quality control of preoperative imaging," Eur. J. Vasc. Endovascular Surg. 23(4), 370-371 (2002).

35. R. W. Boekhoven et al., "A novel experimental approach for threedimensional geometry assessment of calcified human stenotic arteries in vitro," Ultrasound Med. Biol. 39, 1875-1886 (2013).

36. M. Jaeger et al., "Real-time clinical clutter reduction in combined epi-optoacoustic and ultrasound imaging," Photonics Lasers Med. 3(4), 343-349 (2014).

37. M. K. A. Singh and W. Steenbergen, "Photoacoustic-guided focused ultrasound (PAFUSion) for identifying reflection artifacts in photoacoustic imaging," Photoacoustics 3(4), 123-131 (2015).

38. R. W. Boekhoven et al., "Towards mechanical characterization of intact endarterectomy samples of carotid arteries during inflation using echoCT,' J. Biomech. 47, 805-814 (2014).

Mustafa Umit Arabul received his BSc degree in electrical and electronics engineering from Middle East Technical University, Ankara and his MSc degree in biomedical engineering from the Institute of Biomedical Engineering of Bogazici University, Istanbul. He is a PhD candidate at the Eindhoven University of Technology. His current research interests include in vivo photoacoustic imaging of carotid arteries and fundamental characterization and clinical validation of photoacoustic imaging. He is a member of SPIE, NVMU, and IEEE.

Maarten Heres received his MSc degree in biomedical engineering from the University of Twente, The Netherlands. He is a PhD candidate at the Eindhoven University of Technology. His current research interests include the clinical validation of photoacoustics and ultrasound techniques in the assessment of skeletal muscle and skin perfusion. He is a member of the Dutch Society for Medical Ultrasound (NVMU) and IEEE.

Marcel C. M. Rutten received his MSc degree in mechanical engineering from Eindhoven University, Eindhoven, The Netherlands, in 1993. He continued on a PhD project on fluid structure interaction in large arteries, and received his $\mathrm{PhD}$ in 1998, also from the Department of Mechanical Engineering of Eindhoven University of Technology. Since then, he teaches cardiovascular mechanics in the Department of Biomedical Engineering at Eindhoven University.

Marc R. van Sambeek received his medical degree from the Catholic University, Nijmegen, The Netherlands, in 1986 . He is a vascular surgeon at the Catharina Hospital Eindhoven. He was trained as a surgeon in Rotterdam to St. Francis Hospital and the Erasmus Medical Center. He was appointed as staff surgeon at the Erasmus Medical 
Center until 2007. His special focus is on minimally invasive vascular surgery and endovascular treatment.

Frans N. van de Vosse is a professor of cardiovascular biomechanics group of Eindhoven University of Technology (TU/e). He studied applied physics at TU/e from 1976 to 1982 . He earned his PhD in 1987 with his study on the numerical analysis of carotid artery flow. His current research interests are related to the computational and experimental biomechanical analysis of the cardiovascular system and its application to clinical diagnosis and intervention, cardiovascular prostheses, extracorporeal systems, and medical devices.
Richard G. P. Lopata received his BSc and MSc degrees in biomedical engineering from TU/e. He is an assistant professor of the Pulse Lab of Eindhoven University of Technology (TU/e). He earned his PhD from Radboud University Nijmegen Medical Centre with his work on "3D Functional Imaging of the Heart." His current research focuses on the multimodality imaging and medical image analysis (strain imaging, elastography, 2-D and 3-D ultrasound and photoacoustics) in cardiovascular applications. 